\title{
Interacting effect of two social factors on 18-month-old infants' imitative behavior: Communicative cues and demonstrator presence
}

\author{
Krisztina Kupán ${ }^{\mathrm{a}}$, Ildikó Király ${ }^{\mathrm{b}}$, Kinga Kupán ${ }^{\mathrm{c}}$, Kata Krekód ${ }^{\text {, Ádám Miklósia }}{ }^{\mathrm{a}}$, József Topál ${ }^{\mathrm{d}}$ \\ a Department of Ethology, Eötvös Loránd University, Budapest 1117, Hungary \\ b Cognitive Psychology Department, Institute of Psychology, Eötvös Loránd University, \\ Budapest 1064, Hungary \\ c London E14 3UQ, UK \\ d Institute of Cognitive Neuroscience and Psychology, Research Centre for Natural Sciences, \\ Hungarian Academy of Sciences, Budapest 1117, Hungary
}

\begin{abstract}
Certain aspects of a demonstration have been shown to influence infants' interpretation of an observational situation and result in selective imitation. Studying social factors that trigger selective imitation may improve our understanding of how infants encode certain situations. However, only a few studies have investigated the possible interactions among these factors. In our study, 18-month-old infants $(\mathrm{N}=54)$ observed an adult demonstrator retrieve a toy from under an opaque ("baited") container by manipulating another transparent empty one. Infants were assigned to one of four conditions representing all combinations of two social factors: ostensive communication during demonstration (Communicative vs. Noncommunicative) and presence of the demonstrator during reenactment (D-present vs. D-not present). Results suggest that infants' choice behavior was formed in two steps: during the demonstration and during the test phase. Furthermore, an interaction between the effects of the two levels was observed. Communication during the demonstration triggered imitative learning. Infants tended to copy the observed manipulation to learn the communicatively assigned way to reach the goal. This choice behavior was not influenced later by the presence or absence of the demonstrator. The non-communicative demonstration, however, did not elicit a particular learning mechanism.
\end{abstract}

\section{Introduction}

Studying the context-dependent emergence of imitation (or selective imitation [Hilbrink, Sakkalou, Ellis-Davies, Fowler, \& Gattis, 2013; Király, Csibra, \& Gergely, 2013]) in human infants can help us to understand and differentiate possible encoding and choice-making processes behind imitation (Keupp, Behne, Zachow, Kasbohm, \& Rakoczy, 2015; Over \& Carpenter, 2012). The effect of single factors on infant imitation has been studied extensively over the past few years. The following have been shown to have a facilitatory effect on infants' imitative behavior: belonging to the same social group (Buttelmann, Zmyj, Daum, \& Carpenter, 2013), familiarization with the demonstrator (Learmonth, Lamberth, \& RoveeCollier, 2005; Shimpi, Akhtar, \& Moore, 2013), and transient features of the social context such as relational affiliation or friendly behavior of the demonstrator as opposed to an "aloof" person (Nielsen, 2006).

It has also been shown that ostensive communicative cues, such as eye contact and verbal attention getting that signal the demonstrator's communicative intent, may also play a major role in promoting imitative behavior (Király et al., 2013). This finding is in line with the findings obtained in earlier experiments (Brugger, Lariviere, Mumme, \& Bushnell, 2007; Nielsen, 2006), where infants were more likely to imitate actions after having seen the demonstration in a communicative context than in a non-communicative context. Moreover, 
evidence suggests that in the presence of communicative cues infants tend to interpret an action demonstration as a generally accepted way to perform the observed behavior and, therefore, tend to imitate even unusual and inefficient actions (Brugger et al., 2007).

Accordingly, Csibra and Gergely (2009) proposed that communicative demonstrations trigger generalized or normative learning, where the observer considers the entire demonstration to be customary ("the way we do this") and reenacts the observed demonstration in a nonselective manner. This account, however, does not take into consideration the presence or absence of the demonstrator during reenactment.

Only few studies have investigated the possible effects of the presence of the demonstrator during reenactment. Király (2009) found that 1 week after the observation of a successful tool use, a higher percentage of 14-month-old infants imitated the observed action when the demonstrator was present during the reenactment than when she was absent. This suggests that the demonstrator could act as a reminder cue and/or a knowledgeable communicative partner to trigger imitative behavior. In a study by Nielsen and Blank (2011), 4- and 5-yearold children observed two female experimenters performing the same means-action; one of them performed only the necessary steps to reach the goal, whereas the other one integrated some irrelevant steps. Children copied the irrelevant steps only when the demonstrator who performed them was present during the test. These results indicate that children, when imitating a previously observed action, are specifically sensitive to the presence of the particular person from whom the knowledge was obtained. The authors interpreted this demonstrator-selective imitation as children's motivation to promote their shared experience and to build rapport with the demonstrator.

Importantly, the two aforementioned factors affect different stages of the learning process: the communicative cues during demonstration and the presence of the demonstrator during reenactment. Therefore, it stands to reason that these factors affect children's tendency to imitate interacting with each other (Király et al., 2013). However, it is a largely unexplored aspect of infants' imitative behavior, with only a few studies systematically focusing on the interaction between these factors (Hoehl, Zettersten, Schleihauf, Grätz, \& Pauen, 2014; Király et al., 2013). In fact, communicative demonstration was used in all studies where the effect of the presence of the demonstrator during reenactment was investigated (Király, 2009; Király et al., 2013; Nielsen \& Blank, 2011). Furthermore, in studies where the effects of communication were examined, there was some communicative interaction included, at least during some phases of the non-communicative context of the experiment (Brugger et al., 2007; Hoehl et al., 2014; Király et al., 2013; Shimpi et al., 2013).

Here, we investigated how combinations of these factors-(a) the ostensive- communicative manifestation of the demonstrator during demonstration and (b) the presence of the demonstrator during reenactment-affect the imitative behavior of infants. We created a tooluse task in which participants could see how an object is retrieved from under an opaque ("baited") container by the manipulation of a distant and obviously empty (transparent) one. Importantly, the situation was conflicting given that infants could either imitate the observed less efficient action (by following the demonstrator's solution to manipulate the empty container) or emulate the goal by performing a more efficient but self-developed solution (manipulating the baited container).

Based on findings of previous studies, we expected that infants would show a significantly stronger tendency to imitate the demonstrator's less efficient action in those trials where the demonstrator used ostensive communicative demonstration regardless of her presence. Contrarily, in the Noncommunicative/D-not present condition, we expected infants to be more likely to explore alternative (goal-oriented) actions through emulation. In non-communicative contexts, even when a demonstrator was present during reenactment, lower imitation level was found compared with communicative contexts (e.g., Brugger et al., 2007). However, 
other data indicate that children tend to imitate extensively when they try to build or maintain a bond with the demonstrator (Nielsen, 2006; Over \& Carpenter, 2009). Because we excluded all forms of communication from our non-communicative context, we assumed that imitation might appear in the presence of the demonstrator so as to establish a social connection with her. Thus, we expected an interacting effect of the two social factors on infants' imitative behavior.

\section{Method}

\section{Participants}

A total of 54 infants (27 girls and 27 boys, Mage $=18.12$ months, SD $=0.25$, range $=17.5-$ 18.9) were included in the final analysis (see Table S1 in online supplementary material). Participants were selected from a database of volunteering families. Thirteen infants were tested but excluded from the analyses due to parental interference $(n=7)$ or experimenter error $(n=6)$. "Non-responsive" infants, who did not make a choice within $90 \mathrm{~s}$, were also excluded $(n=10)$. Infants were assigned randomly to one of four experimental conditions.

\section{Experimental arrangement}

Participants were tested at the baby lab $\left(5 \_5 \mathrm{~m}\right)$ of the Institute of Cognitive Neuroscience and Psychology, Hungarian Academy of Sciences. A transparent plastic container and an opaque plastic container of equal shape and size $(12 \mathrm{~cm}$ high and $16 \mathrm{~cm}$ in diameter) were placed $0.6 \mathrm{~m}$ apart on the floor. Both were turned with their openings down and placed on a black platform (15 _ $15{ }_{-} 6 \mathrm{~cm}$ ). The white opaque container was used to hide the target object (a tennis ball). The platform was slightly aslope so that the ball rolled out when the baited container was elevated. The two containers were connected by a string, which was led through two pulleys fixed to the ceiling. A curtain covered the pulleys, and this was also used to prevent the infants from witnessing the rebaiting of the container. By moving the empty container horizontally, the baited container lifted up (see Figs. S1 and S2 in supplementary material). Infants were videotaped by two cameras (one facing the infant and one facing the experimental apparatus).

\section{Procedure}

Familiarization phase (10-15 min)

Right after entering, the mother sat down while the infant was allowed to explore the room and play with toys. A female experimenter (helper) talked to and played with the infant. The demonstrator (the first author) was also present and occasionally smiled at the baby and talked to the mother; however, she neither talked to nor played with the infant. Following the familiarization, the toys were put away. A curtain covered the experimental apparatus throughout this phase.

\section{Demonstration phase}

The parent was asked to sit down on a chair facing the containers (at a distance of $3 \mathrm{~m}$ ) and hold the infant on her lap. The demonstrator placed the ball under the opaque container, while the curtain prevented the infant from witnessing the baiting procedure. Infants witnessed the demonstrations in one of the following conditions. 
Communicative demonstration. The demonstrator, who was standing between the two containers, pulled back the curtains and looked at the infant. She addressed the infant by name and said, "Look! I am going to show you something interesting! We are going to play with a tennis ball!" After that, she touched the two containers simultaneously. Then, the demonstrator addressed the infant again ("Look at this!"). As the demonstrator made eye contact with the infant, she grasped the empty container by both hands and placed it on the ground ahead of the platform. At that point, the baited container elevated and the tennis ball rolled out toward the infant. Then, the demonstrator turned her head toward the ball, shifting her gaze conspicuously from the infant to the ball. Finally, she picked up the ball and bounced it twice to the ground but did not give it to the infant. She closed the curtain and re-baited the opaque container.

Non-communicative demonstration. This procedure was identical to that described above except that the demonstrator turned her back to the infant and performed the actions without ostensive communicative signals. She did not look at the infant, nor did she address or instruct the infant. During the entire procedure, the demonstrator mumbled a short meaningless poem to non-communicatively attract the infant's attention to the demonstration. In each condition, the action demonstration was repeated a total of three times. The side of the baited container was counterbalanced between participants.

Test phase

Immediately after the third demonstration, the demonstrator closed the curtain and placed the ball under the opaque container. Then, she pulled back the curtains again and either left the room (D-not present condition) or remained there (D-present condition).

D-present condition. After communicative demonstration, the demonstrator stood motionless at a predetermined

place (on the left side, about halfway between the participant and the apparatus) and

encouraged the participant by looking at him or her and saying, “Now it’s your turn!”. After noncommunicative

demonstration, however, the demonstrator sat on a chair (where she stood in the

communicative demonstration) with her back half-turned to the apparatus while reading a newspaper.

In this case, she neither looked at nor talked to the infant.

D-not present condition. In this situation, the demonstrator left the room without talking to the infant. After communicative demonstration, she looked and smiled at the infant once before leaving the room. Following the demonstration, the mother released her infant, who was then encouraged to explore the apparatus ("Now it’s your turn! You can try it!"). Importantly, the parent was not allowed to make comments, give verbal instructions, or point toward the apparatus. The infant had $90 \mathrm{~s}$ to approach and manipulate the apparatus.

\section{Coding and data analyses}

Infants' "first choice” was recorded. A container was regarded as chosen if infants touched it with their hand, manipulated it, or at least unambiguously pointed at it. The manipulation was further analyzed (see Tables S2 and S3 in supplementary material). Based on the container choice (empty or baited) and the manipulation they performed, infants were categorized into one of three response categories. In the "emulative response" (0) category, the infants approached and manipulated the baited container. In the "pseudo-imitative response" (1) category, the infants approached and manipulated the empty container, but the infants' behavior did not contain any elements of the demonstrator's manipulation. We created this 
variable to differentiate between two behavioral forms that potentially reflect differences in the understanding of the original demonstration. Manipulating the target location without any elements of the original means-actions could be a result of local enhancement (Want \& Harris, 2002) or another low-level social learning mechanism. In the “imitative response” (2) category, the infants approached and manipulated the empty container and copied some elements of the way the demonstrator had performed the action (for more details, see Tables S2 and S3).

For those infants who performed imitative or pseudo-imitative responses, we also recorded whether they showed any interest in the potential effect of their manipulation. This "causality checking” behavior (cf. Carpenter, Akhtar, \& Tomasello, 1998) was introduced to show that infants encoded that the means they reenact is indeed the way to arrive at the observed outcome. This additional variable allowed us to confirm differentiation of potential underlying learning formats; it suggests insightful social learning when causality checking occurs after manipulating the empty container, whereas its absence indicates a lower level learning. Causality checking was defined as showing one or more of the following three actions: (a) orientation to the baited container while manipulating the empty one, (b) switching to the baited container immediately after manipulating the empty one, and (c) getting the ball immediately after manipulating the empty container (Table S2).

To assess inter-observer agreement, a second person blind to the demonstration scored the data. Cohen's j values were .90 (for manipulation categories) and .91 (for causality checking), showing a high level of reliability.

Statistical analyses were conducted with R 3.1.1 (R Development Core Team, 2014). Choice behavior was analyzed with non-parametric ordinal logistic regression model. This model presumes a nonparametric response variable on an ordinal scale. Our response variable had three levels $(0,1$, and 2$)$ where the following values referred to closer similarity to the demonstrated act. Our data fit all preassumptions of the model. The effects of "demonstration type" and "presence of D" as main factors and their interaction were tested in the model. Post hoc tests were carried out by Mann-Whitney U tests. Due to multiple comparisons levels of significance ( $p$ values), we used false discovery adjustment for correction of the data after Benjamini and Hochberg (1995). Causality checking (present vs. non-present) was analyzed by binomial tests (test proportion $=.50$ ).

\section{Results}

Ordinal logistic regression analysis on the two main factors (demonstration type and presence of D) revealed significant effects of both factors on infants' choice behavior (demonstration type: coefficient value $=4.02, \mathrm{p}=.0001$, adjusted $\mathrm{a}=.014$; presence of $\mathrm{D}$ : coefficient value $=$ $2.49, \mathrm{p}=.007$, adjusted $\mathrm{a}=.036$ ). The interaction of the two main factors was significant (interaction coefficient value $=\_3.41, \mathrm{p}=.007$, adjusted $\mathrm{a}=.029$ ). Post hoc analyses revealed no significant differences when comparing the Communicative/D-present and Communicative/D-not present conditions, $\mathrm{U}(1)=108.5, \mathrm{p}=.30$, adjusted $\mathrm{a}=.043$. There was no difference between the Communicative/D-present and Non-communicative/D-present conditions either, $\mathrm{U}(1)=76.0, \mathrm{p}=.44$, adjusted $\mathrm{a}=.05$ (Fig. 1 ).

However, differences were observed between the Non-communicative/D-present and Noncommunicative/D-not present conditions, $\mathrm{U}(1)=39.0, \mathrm{p}=.005$, adjusted $\mathrm{a}=.021$, as well as between the Communicative/D-not present and Non-communicative/D-not present conditions, $\mathrm{U}(1)=16.0, \mathrm{p}<.001$, adjusted $\mathrm{a}=.007$. These differences were driven by an increase in the emulative responses (more frequent selection of the baited container) in the Noncommunicative/D-not present condition compared with the other conditions. Specifically, the 
number of responses in the two "empty choice" response categories (i.e., imitation and pseudo-imitation) decreased in this condition.

Causality checking behavior was relatively rare (33\%) among those infants who performed pseudoimitative responses (binomial test, $\mathrm{p}=.50$ ). However, $89 \%$ of infants who imitated some elements of the demonstrator's action while manipulating the empty container performed causality checking behavior (binomial test, $\mathrm{p}<.001$ ) (Table 1).

Figure 1. Number and percentage of infants exhibiting "emulative” (0), "pseudo-imitative" (1), and "imitative” (2) responses in each of four experimental conditions.

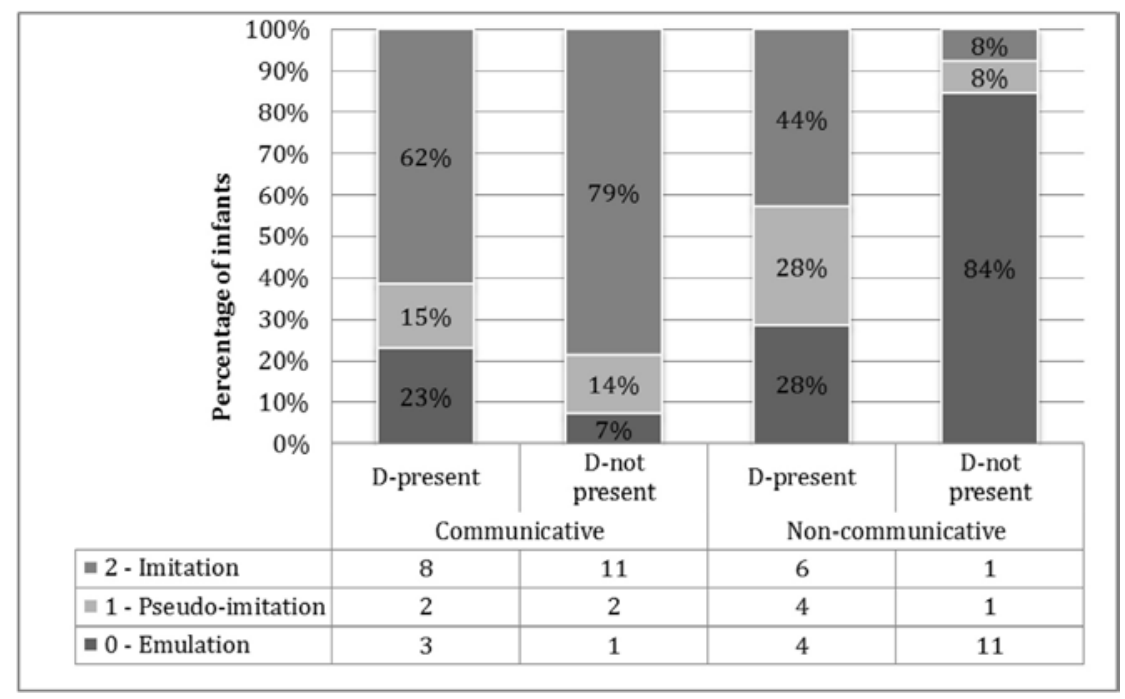

Table 1 Number of infants who performed "causality checking” out of all infants in each experimental group.

\begin{tabular}{lll} 
Experimental condition & Pseudo-imitation & Imitation \\
Communicative/D-present & $2 / 2(100)$ & $7 / 8(88)$ \\
Communicative/D-not present & $0 / 2(0)$ & $9 / 11(82)$ \\
Non-communicative/D-present & $1 / 4(25)$ & $6 / 6(100)$ \\
Non-communicative/D-not present & $0 / 1(0)$ & $1 / 1(100)$ \\
\hline Total & $3 / 9(33)$ & $23 / 26(89)$
\end{tabular}

Note. The two "empty choice" response categories (imitative and pseudo-imitative) are depicted. Percentages of infants who

performed causality checking are in parentheses.

\section{Discussion}

Our purpose in the current study was to investigate the possible co-effect of a communicatively accentuated action demonstration and the presence or absence of the demonstrator during reenactment on the imitative behavior of infants. Previous studies showed effects of both social contextual factors (Király, 2009; Király et al., 2013; Nielsen \& Blank, 2011). However, the possibility that these factors influence each other's effect was not tested. The design of our task made it possible to differentiate between imitative and emulative responses because the less effective manipulation was spatially separated from the location of the goal object. 
During the demonstration phase the ball emerged from under the baited container due to the demonstrator's manipulation of the empty one. Infants in this situation could follow one of three strategies: (a) goal-oriented emulation, that is, approaching the opaque baited container (a self-developed efficient solution); (b) pseudo-imitation, that is, approaching the empty container without repeating any action of the demonstrator; or (c) imitation, that is, approaching the empty container and reenacting one or more elements of the demonstrator's actions.

In addition, we tested infants' understanding of the causality between the action manipulation and the outcome (i.e., causality checking; Carpenter et al., 1998). Our findings confirm that infants copy the observed action in order to reach the observed result, which is an assumption of insightful imitation. In fact, significantly fewer infants checked the baited container while manipulating the empty one in the pseudo-imitative response group (33\%) than in the imitative response group (89\%) (see Table S3 in supplementary material). Using this strategybased categorization, we compared the choice behavior of infants between the different conditions and found that the two social contextual factors interactively influence infants' choice behavior.

Due to the use of ostensive-communicative cues during the demonstration, the majority of infants performed imitative responses independently of the presence or absence of the demonstrator during reenactment $(62 \%$ and $79 \%$, respectively). This suggests that communicative cues have a stronger effect on infants' choice behavior than the presence or absence of the demonstrator. The response was already formed during the demonstration phase and was not further modified during the test phase. This finding is in line with our hypothesis and further confirms the assumption that ostensive-communication has the potential to improve the encoding of the particular means that the demonstrator used to reach the goal (Gergely \& Csibra, 2006; Király et al., 2013). Furthermore, such communication may also enhance infants' tendency to repeat what they observed in order to achieve the goal in a "socially prescribed" way while disregarding the efficiency of the solution. This interpretation could serve as a basis for social instrumental or normative learning (Csibra \& Gergely, 2009; Kenward, 2012).

In contrast, infants' behavior in the non-communicative context was strongly affected by the presence of the demonstrator. This suggests that the non-communicative demonstration did not robustly evoke a particular learning mechanism and the final choice behavior was formed during the encoding phase. When the demonstrator was absent during the test phase, most infants (84\%) produced an emulative response. In the absence of social influence, infants may have relied more heavily on their interpretation of the situation and developed a more efficient solution to reach the observed goal (goal emulation; see Brugger et al., 2007; Nielsen, 2006). The Non-communicative/D-present situation, however, appears to have failed to promote any one particular strategy. Visual inspection of the data indicates that infants performed more imitation in the Non-communicative/D-present situation relative to the D-not present condition. However, infants' responses were relatively evenly distributed across the three response categories (imitative response: 44\%; pseudo-imitative response: 28\%; emulative response: 28\%). Of note, it was in this condition that the greatest number of infants needed to be excluded due to non-responsiveness (5/10; see Table S1 in supplementary material).

Visual inspection of the data further shows that infants imitated in the absence of the demonstrator only after a communicative context but not in the non-communicative context (79\% vs. 8\%). This further confirms the importance of the communicative demonstration in facilitating imitative tendencies (Király et al., 2013). Our results suggest that in a noncommunicative situation goal emulation becomes a dominant tendency only when infants are free from the demonstrator's social influence during reenactment. 
In general, we found no difference between the Communicative/D-present and Noncommunicative/D-present conditions. However, the motivations underlying the apparent imitative behavior were likely different between the two conditions. In the communicative situation, communicative cues are presumably the main influencing factors and the presence of the demonstrator has no further effect on choice behavior. The presence of the demonstrator during reenactment after a non-communicative demonstration, however, diverts infants from their self-developed, more efficient (emulative) solution, presumably by offering alternative interpretations. Nevertheless, the role of the demonstrator as an influencing factor is unclear. Király (2009) suggested that the demonstrator during reenactment can act as a reminder cue in two ways through prompting different functions: by prompting an initiation of communication and by reminding the child of being engaged in a social context and, thus, inducing social pressure. In our Non-communicative/D-present situation, the infant could have tried to establish a communicative relationship with the demonstrator through an imitative act (Nielsen, 2006). Alternatively, the mere presence of the other partner exerted social pressure, which has been described as a strong motive for an individual to act like the other members of his or her group to “fit in” (Over and Carpenter, 2012).

In conclusion, the current study provides the first evidence for the combined effect of ostensive communicative demonstration and the demonstrator's presence during reenactment on 18-monthold infants' choice behavior. Imitative responses may represent different motives in the different contexts; while ostensive communicative demonstration triggers learning about the observed action, the presence of the demonstrator in a non-communicative context is more likely to elicit a tendency to conform to the demonstrator's behavior. The exact role that the demonstrator's presence plays in the emergence of imitative, pseudo-imitative, or emulative responses is still unclear and, thus, is worthy of further investigation.

\section{Acknowledgments}

This work was funded by the Hungarian Science Foundation (OTKA K112138). We thank György Gergely for his comments and suggestions and thank Tímea Vándor for her assistance in data collection. We are grateful to Nóra Bunford for reviewing the language of the manuscript.

\section{Supplementary material}

Supplementary data associated with this article can be found, in the online version, at http://dx.doi.org/10.1016/j.jecp.2017.03.019.

\section{References}

Benjamini, Y., \& Hochberg, Y. (1995). Controlling the false discovery rate: A practical and powerful approach to multiple testing. Journal of the Royal Statistical Society B: Methodological, 57, 289-300.

Brugger, A., Lariviere, L. A., Mumme, D. L., \& Bushnell, E. W. (2007). Doing the right thing: Infants' selection of actions to imitate from observed event sequences. Child Development, 78, 806-824.

Buttelmann, D., Zmyj, N., Daum, M., \& Carpenter, M. (2013). Selective imitation of in-group over out-group members in 14-month-old infants. Child Development, 84, 422-428.

Carpenter, M., Akhtar, N., \& Tomasello, M. (1998). Fourteen- through 18-month-old infants differentially imitate intentional and accidental actions. Infant Behavior and Development, 21, 315-330. 
Csibra, G., \& Gergely, G. (2009). Natural pedagogy. Trends in Cognitive Sciences, 13, 148153.

Gergely, G., \& Csibra, G. (2006). Sylvia's recipe: The role of imitation and pedagogy in the transmission of cultural knowledge. In N. J. Enfield \& S. C. Levenson (Eds.), Roots of human sociality: Culture, cognition, and human interaction (pp. 229-255). Oxford, UK: Berg.

Hilbrink, E. E., Sakkalou, E., Ellis-Davies, K., Fowler, N. C., \& Gattis, M. (2013). Selective and faithful imitation at 12 and 15 months. Developmental Science, 16, 828-840.

Hoehl, S., Zettersten, M., Schleihauf, H., Grätz, S., \& Pauen, S. (2014). The role of social interaction and pedagogical cues for eliciting and reducing overimitation in preschoolers. Journal of Experimental Child Psychology, 122, 122-133.

Kenward, B. (2012). Over-imitating preschoolers believe unnecessary actions are normative and enforce their performance by a third party. Journal of Experimental Child Psychology, 112, 195-207.

Keupp, S., Behne, T., Zachow, J., Kasbohm, A., \& Rakoczy, H. (2015). Over-imitation is not automatic: Context sensitivity in children's overimitation and action interpretation of causally irrelevant actions. Journal of Experimental Child Psychology, 130, 163-175.

Király, I. (2009). The effect of the model's presence and of negative evidence on infants' selective imitation. Journal of Experimental Child Psychology, 102, 14-25.

Király, I., Csibra, G., \& Gergely, G. (2013). Beyond rational imitation: Learning arbitrary means actions from communicative demonstrations. Journal of Experimental Child Psychology, 116, 471-486.

Learmonth, A. E., Lamberth, R., \& Rovee-Collier, C. (2005). The social context of imitation in infancy. Journal of Experimental Child Psychology, 91, 297-314.

Nielsen, M. (2006). Copying actions and copying outcomes: Social learning through the second year. Developmental Psychology, 42, 555-565.

Nielsen, M., \& Blank, C. (2011). Imitation in young children: When who gets copied is more important than what gets copied. Developmental Psychology, 47, 1050-1053.

Over, H., \& Carpenter, M. (2009). Priming third-party ostracism increases affiliative imitation in children. Developmental Science, 12, F1-F8.

Over, H., \& Carpenter, M. (2012). Putting the social into social learning: Explaining both selectivity and fidelity in children's copying behavior. Journal of Comparative Psychology, 126, 182-192.

R Development Core Team (2014). R: A language and environment for statistical computing. Vienna, Austria: R Foundation for Statistical Computing.

Shimpi, P. M., Akhtar, N., \& Moore, C. (2013). Toddlers' imitative learning in interactive and observational contexts: The role of age and familiarity of the model. Journal of Experimental Child Psychology, 116, 309-323.

Want, S. C., \& Harris, P. L. (2002). How do children ape? Applying concepts from the study of non-human primates to the developmental study of "imitation" in children. Developmental Science, 5, 1-14. 